\title{
BEYOND INDOOR PRESENCE MONITORING WITH SIMPLE SENSORS
}

\author{
Tuan Anh Nguyen and Marco Aiello \\ Distributed Systems Group, University of Groningen, The Netherlands \\ \{t.a.nguyen,m.aiello\}@rug.nl
}

Keywords: Context-Awareness; Activity Recognition; Energy-Awareness; Wireless Sensor Networks

Abstract: To have buildings that are able to adapt to the user needs and at the same time to operate efficiently, it is essential to know the activity the people are performing. Presence sensors, which are widely deployed in modern buildings, attempt to regulate lighting to the presence of people in indoor spaces. Though, much more in terms of comfort and energy efficiency can be achieved if more detailed information on the activity of the users is detected. In this paper, we provide an initial investigation on detecting indoor activities by using simple sensors (infrared, pressure and acoustic) deliberately avoiding the use of rich sensors such as cameras. The sensors are low-cost, wireless, and retrofittable in existing structures. Our prototype is able to recognize five activities (working at a desk with or without a PC, having a meeting, and the presence/absence in the office) with accuracy of almost $95 \%$, while unaffecting user's behavior and comfort.

\section{INTRODUCTION}

Buildings account for more than $40 \%$ of energy consumption in EU-27, with up to $50 \%$ in the UK and Switzerland, and are the largest $\mathrm{CO} 2$ producers (EPBD, 2002). In addition, for typical industrial and commercial buildings power usage amounts to roughly $30 \%$ of the total operational costs. Hence, towards a low carbon economy, making "smarter" use of energy in buildings will fundamentally contribute to energy and cost savings. Building automation systems (BAS) provide automatic control of the conditions of indoor environments. Their primary goal is to realize significant savings in energy and reduce cost. While BAS can currently operate lighting and heating, ventilating and air conditioning (L-HVAC) systems efficiently, they are based on very simple solutions to detect the presence of people. In fact, current technology is based on infrared sensors that detect movement and assume presence for a fixed temporal interval after the last movement was detected. Such a solution is reasonable in first approximation, but clearly having better understanding of user activities, would lead to better control and possibly higher user comfort.

User activity is an essential ingredient for maximizing the energy savings and spurring rapid adoption in buildings. The recognition solutions should be very accurate to satisfy the user and provide effec- tive energy savings, while consuming not too much energy itself to function nor invading the privacy of the user. Most current solutions for BAS still operate based on pre-determined schedules. HVAC condition rooms assuming maximum occupancy rather than actual usage. Most occupancy sensors currently installed within buildings are fairly coarse-grained and inaccurate. Passive infrared (PIR) based sensors are often used for occupancy given their relative low cost and energy consumption, though these sensors only sense movements and most of them use a timeout for determining room occupancy. More advanced systems have been deployed using cameras and vision algorithms, but these systems suffer from deployability, cost and privacy issues.

We propose an accurate activity recognition system working at the level of individual offices. Our work contributes to one of the key components of an energy-aware framework based on embedded service middleware and a building-distributed architecture of smart objects. The platform is currently under development within the European Framework 7 project GreenerBuildings, (GreenerBuildings, 2010). In the present work, we design and illustrate a system based on a battery-based wireless sensor network that can identify five activities of one person in an office, possibly with other people present in the room (e.g, meeting at the table). The activities are: working at a desk with or without a PC, having a meeting, and the pres- 
ence/absence in the office, shown in Table 1 This is done combining sensed data from a PIR, pressure and acoustic sensors. We evaluate the initial prototype in terms of accuracy and show that it can detect the activities in single room offices. We stress the requirement of not resorting to any advanced sensors which are expensive or require a change in user behavior, such as cameras, RFID tags, or wearable sensors. Instead of that, simple, wireless, binary sensors are enough for accurate activity recognition. Through a number of experiments performed in our offices, we show that with the proposed solution it is possible to recognize five activities of interest with $94.81 \%$ accuracy.

The remainder of the paper is organized as follows. In Section 2, we describe the requirements including the desired set of recognized activities. Section 3 presents the implementation of the solution in an actual office and experimental results. We compare our approach with related work in Section 4 Finally, we conclude by summing up our findings and propose future direction for investigation in Section 5

\section{INDOOR ACTIVITY RECOGNITION}

The main requirements for performing indoor activity recognition with the goal of providing input to a control strategy for energy savings in office buildings are the following ones.

- (Rl) Discriminative. The solution should distinguish as many activities as possible which are typical of office presence.

- (R2) Simple sensors. The sensors used in the solution should be inexpensive and easy to retrofit in existing buildings.

- (R3) Energy parsimonious. The sensing solution should itself consume not too much energy.

- (R4) Privacy preserving. The solution should be as less invasive as possible and affect the user privacy as little as possible.

- (R5) Accurate. The solution should be accurate in its recognition of user activities.

\subsection{Requirement Analysis}

We address (R1) by performing a survey for a complete working day ( 8 hours) in two offices, one at a higher education institution's office building in Groningen (Hanzehogeschool, The Netherlands) used by two staff members and the another one at the

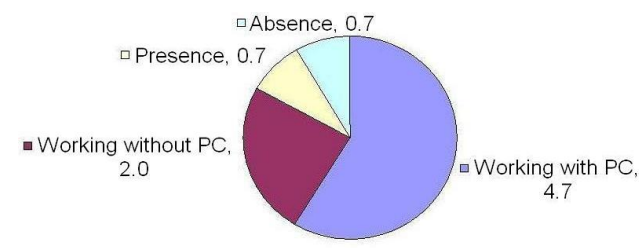

Mennes \& Jager in Groningen office

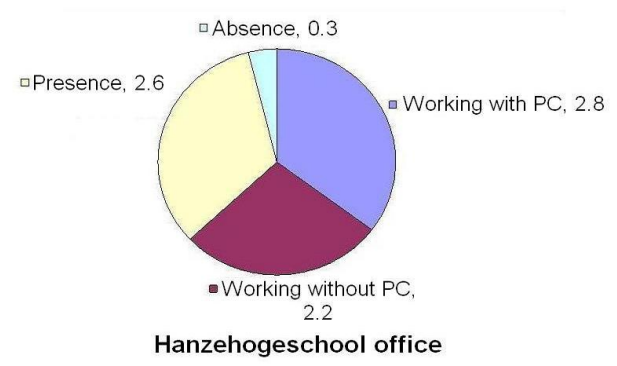

Figure 1: Activities of interest survey results

premises of Mennes \& Jager in Groningen, an electronics company with five staff members. The offices were filmed and then the footage used to classify by hand the activities of people over time. The results of the manual classification are summarized in Figure 1 as pie-charts. One notices in both cases a dominance of being at the desk working with a PC or without one. At both Mennes \& Jager and Hanzehogeschool, occupants mainly spend their time working with computers at their desks, nearly 5 hours and 3 hours, respectively. They spend the main part of their remaining time sitting on their chairs at the desk doing other tasks. In total, staff at Hanzehogeschool use 5 hours $(63 \%)$ out of their 8-hour working day sitting at their desk versus 6.7 hours (84\%) at Mennes \& Jager. Absence is considered as an activity, representing the unoccupied status of the office. Both offices are not occupied more or less for a half hour during the filmed working day. Presence indicates that user is in the room but the specific activity does not have any significant impact on changing the state of the appliances. Activities of this kind include temporarily walking or standing looking through windows, drinking tea and so on. In the data collection at Hanzehogeschool, presence happens in two hours and a half while the same occurs for just 0.7 hour at Mennes \& Jager in Groningen office. With respect to energy-awareness, "having a meeting" is an activity that requires appliance's adaptations such as turning the lights on during the meeting and turning them off otherwise, thus this activity is worth taking into account in order to save energy and satisfy user comfort. Therefore, in first instance we take the activities that have emerged from the video study as the starting point of the indoor activity recognition system; namely: 1) Working with 
PC, 2) Working without PC, 3) Having a meeting, 4) Presence, and 5) Absence. Activities and their definition are described in Table 1

Table 1: Office activities

\begin{tabular}{|l|l|}
\hline Activity & Definition \\
\hline Activity 1: Working with PC & Manipulating keyboard or mouse \\
\hline Activity 2: Working without PC & $\begin{array}{l}\text { Sitting at the desk but not manipulat- } \\
\text { ing keyboard and mouse }\end{array}$ \\
\hline Activity 3: Having a meeting & Discussing at the meeting table \\
\hline Activity 4: Presence & $\begin{array}{l}\text { Being active in the room but the spe- } \\
\text { cific activity is not recognized }\end{array}$ \\
\hline Activity 5: Absence & Being absent from the room \\
\hline
\end{tabular}

The requirements (R2)-(R4) are strictly interdependent as they mostly involve the choice of the type of sensor and the characteristics of the hardware currently available that implements them. We need to avoid cameras for privacy reasons and sensors which require users to wear them, such as RFID, active badges and the like. We also have to look at sensors which provide discriminative data without costing too much and consuming excessive amounts of energy. In summary, we resort to a combination of anonymous and binary wireless sensors that are cheap, easy to install, require minimal maintenance and supervision, and do not have to be worn or carried, such as 1) chair pressure sensor, 2) acoustic sensor, and 3) passive infrared (PIR) motion sensor. These sensors are placed so that they are able to sense crucial information. In addition, user privacy is unaffected as information is sensed in binary manner (i.e. TRUE, FALSE), for example whether the user is sitting on a chair or not, whether the keyboard/mouse is being operated or not, etc.

Finally, with respect to (R5), we propose the following recognition cases:

- Working with PC activity: we define this activity when the user sits on the working chair AND uses the PC by operating the keyboard/mouse.

- When user sits on a chair but does not operate the keyboard/mouse then user is Working without PC.

- Having a meeting is indicated by user sits on a chair at the meeting table AND speaking.

- Our system reports the current activity is Presence in two cases: when user does not sit on working chair, however user might be sitting on a chair at the meeting table but no discussion is detected. In the second case, no chair in the room is occupied, but movement is still detected inside the room.

- The last activity Absence is indicated if following condition is satisfied: no chair in the room is occupied AND no movement is detected.
Table 2: Activities associated with sensors' values

\begin{tabular}{|c|c|c|c|c|c|c|}
\hline \multirow{2}{*}{ No. } & \multicolumn{5}{|c|}{ Boolean value of sensors } & \multirow{2}{*}{ Activity } \\
\hline & $\begin{array}{l}\text { Working } \\
\text { chair }\end{array}$ & $\begin{array}{l}\text { Key/Mouse } \\
\text { acoustic }\end{array}$ & $\begin{array}{l}\text { Meeting } \\
\text { chair }\end{array}$ & $\begin{array}{l}\text { Meeting } \\
\text { acoustic }\end{array}$ & PIR & \\
\hline 1 & $\mathrm{~T}$ & $\mathrm{~T}$ & $\mathrm{~T}$ & $\mathrm{~T}$ & $\mathrm{~T}$ & \multirow{8}{*}{$\begin{array}{l}\text { Working } \\
\text { with PC }\end{array}$} \\
\hline 2 & $\mathrm{~T}$ & $\mathrm{~T}$ & $\mathrm{~T}$ & $\mathrm{~T}$ & $\mathrm{~F}$ & \\
\hline 3 & $\mathrm{~T}$ & $\mathrm{~T}$ & $\mathrm{~T}$ & F & $\mathrm{T}$ & \\
\hline 4 & $\mathrm{~T}$ & $\mathrm{~T}$ & $\mathrm{~T}$ & F & $\mathrm{F}$ & \\
\hline 5 & $\mathrm{~T}$ & $\mathrm{~T}$ & $\mathrm{~F}$ & $\mathrm{~T}$ & $\mathrm{~T}$ & \\
\hline 6 & $\mathrm{~T}$ & $\mathrm{~T}$ & $\mathrm{~F}$ & $\mathrm{~T}$ & $\mathrm{~F}$ & \\
\hline 7 & $\mathrm{~T}$ & $\mathrm{~T}$ & F & $\mathrm{F}$ & $\mathrm{T}$ & \\
\hline 8 & $\mathrm{~T}$ & $\mathrm{~T}$ & $\mathrm{~F}$ & F & $\mathrm{F}$ & \\
\hline 9 & $\mathrm{~T}$ & $\mathrm{~F}$ & $\mathrm{~T}$ & $\mathrm{~T}$ & $\mathrm{~T}$ & \multirow{8}{*}{$\begin{array}{l}\text { Working } \\
\text { without PC }\end{array}$} \\
\hline 10 & $\mathrm{~T}$ & F & $\mathrm{T}$ & $\mathrm{T}$ & $\mathrm{F}$ & \\
\hline 11 & $\mathrm{~T}$ & F & $\mathrm{T}$ & $\mathrm{F}$ & $\mathrm{T}$ & \\
\hline 12 & $\mathrm{~T}$ & F & $\mathrm{T}$ & F & $\mathrm{F}$ & \\
\hline 13 & $\mathrm{~T}$ & F & $\mathrm{F}$ & $\mathrm{T}$ & $\mathrm{T}$ & \\
\hline 14 & $\mathrm{~T}$ & $\mathrm{~F}$ & $\mathrm{~F}$ & $\mathrm{~T}$ & $\mathrm{~F}$ & \\
\hline 15 & $\mathrm{~T}$ & $\mathrm{~F}$ & $\mathrm{~F}$ & F & $\mathrm{T}$ & \\
\hline 16 & $\mathrm{~T}$ & $\mathrm{~F}$ & F & F & $\mathrm{F}$ & \\
\hline 17 & F & $\mathrm{T}$ & $\mathrm{T}$ & $\mathrm{T}$ & $\mathrm{T}$ & \multirow{4}{*}{$\begin{array}{l}\text { Having } \\
\text { a meeting }\end{array}$} \\
\hline 18 & F & $\mathrm{T}$ & $\mathrm{T}$ & $\mathrm{T}$ & $\mathrm{F}$ & \\
\hline 19 & F & $\mathrm{F}$ & $\mathrm{T}$ & $\mathrm{T}$ & $\mathrm{T}$ & \\
\hline 20 & F & $\mathrm{F}$ & $\mathrm{T}$ & $\mathrm{T}$ & $\mathrm{F}$ & \\
\hline 21 & $\mathrm{~F}$ & $\mathrm{~T}$ & $\mathrm{~T}$ & F & $\mathrm{T}$ & \multirow{8}{*}{ Presence } \\
\hline 22 & F & $\mathrm{T}$ & $\mathrm{T}$ & F & $\mathrm{F}$ & \\
\hline 23 & F & $\mathrm{T}$ & $\mathrm{F}$ & $\mathrm{T}$ & $\mathrm{T}$ & \\
\hline 24 & $\mathrm{~F}$ & $\mathrm{~T}$ & $\mathrm{~F}$ & F & $\mathrm{T}$ & \\
\hline 25 & F & $\mathrm{F}$ & $\mathrm{T}$ & F & $\mathrm{T}$ & \\
\hline 26 & F & $\mathrm{F}$ & $\mathrm{T}$ & F & $\mathrm{F}$ & \\
\hline 27 & F & $\mathrm{F}$ & $\mathrm{F}$ & $\mathrm{T}$ & $\mathrm{T}$ & \\
\hline 28 & F & $\mathrm{F}$ & $\mathrm{F}$ & $\mathrm{F}$ & $\mathrm{T}$ & \\
\hline 29 & F & $\mathrm{F}$ & $\mathrm{F}$ & F & $\mathrm{F}$ & \multirow{4}{*}{ Absence } \\
\hline 30 & F & $\mathrm{T}$ & $\mathrm{F}$ & $\mathrm{T}$ & $\mathrm{F}$ & \\
\hline 31 & $\mathrm{~F}$ & $\mathrm{~T}$ & $\mathrm{~F}$ & $\mathrm{~F}$ & $\mathrm{~F}$ & \\
\hline 32 & $\mathrm{~F}$ & $\mathrm{~F}$ & $\mathrm{~F}$ & $\mathrm{~T}$ & $\mathrm{~F}$ & \\
\hline
\end{tabular}

\subsection{Activity Recognition}

The task of recognizing an activity is reduced to reading the sensor data and associating the data to a specific configuration which, in turn, identifies an activity. This is best represented as a truth table of basic sensor values, shown in Table 2, in which $\mathrm{T}$ means TRUE and F stands for FALSE. The table is exhaustive of all possible readings of the sensors.

The recognition of the described cases are performed on a base station which makes a clas- 
Table 3: Information Fusion at the Base Station

\begin{tabular}{|c|c|}
\hline $\begin{array}{l}\text { Activity 1: } \\
\text { Working with PC }\end{array}$ & $\begin{array}{c}\text { condition }-1=(\text { WorkingChairOccupy }==T R U E \wedge \\
\text { Key/MouseOccupy }==T R U E)\end{array}$ \\
\hline $\begin{array}{l}\text { Activity 2: } \\
\text { Working without PC }\end{array}$ & $\begin{array}{c}\text { condition }-2=(\text { WorkingChairOccupy }==T R U E \wedge \\
\text { Key } / \text { MouseOccupy }==\text { FALSE })\end{array}$ \\
\hline $\begin{array}{l}\text { Activity } 3 \text { : } \\
\text { Having a meeting }\end{array}$ & $\begin{array}{l}\text { condition }-3=(\text { WorkingChairOccupy }==\text { FALSE } \wedge \\
\text { MeetingChairOccupy }==T R U E \wedge \text { HumanVoice }==\text { TRUE })\end{array}$ \\
\hline $\begin{array}{l}\text { Activity 4: } \\
\text { Presence }\end{array}$ & $\begin{array}{l}\text { condition }-4=((\text { WorkingChairOccupy }==F A L S E \wedge \\
\text { MeetingChairOccupy }==T R U E \wedge \text { HumanVoice }==F A L S E) \vee \\
(\text { WorkingChairOccupy }==F A L S E \wedge \text { MeetingChairOccupy }==F A L S E \wedge \\
P I R==T R U E))\end{array}$ \\
\hline $\begin{array}{l}\text { Activity 5: } \\
\text { Absence }\end{array}$ & $\begin{array}{l}\text { condition }-5=(\text { WorkingChairOccupy }==F A L S E \wedge \\
\text { MeetingChairOccupy }==F A L S E \wedge P I R==F A L S E)\end{array}$ \\
\hline
\end{tabular}

sification every minute. Each minute, the Base Station receives reports from the sensors, it fuses the information to decide on what activity is currently occurring. Table 3 shows the information fusion process for recognizing the current activity. In the first case, Activity 1-Working with $P C$ is confirmed by the condition: (WorkingChairOccupy == TRUE $\wedge$ Key/MouseOccupy $==$ TRUE). Activity 2-Working without $P C$ is recognized through the change in Keyboard/Mouse Occupy status, with the condition: (WorkingChairOccupy == TRUE $\wedge$ Key/MouseOccupy = FALSE). Acoustic sensor at the meeting table is used to check the status of Human voice appearance and $A c$ tivity 3-Having a meeting is recognized under the condition: (WorkingChairOccupy $==$ FALSE $\wedge$ MeetingChairOccupy $==$ TRUE $\wedge$ HumanVoice $==T R U E$ ). In the case of Activity 4-Presence the following condition is checked: ((MeetingChairOccupy $==$ TRUE $\wedge$ HumanVoice $=$ FALSE) $\bigvee$ (WorkingChairOccupy $==$ FALSE $\wedge$ MeetingChairOccupy $==$ FALSE $\wedge P I R==$ TRUE). Finally, (WorkingChairOccupy $==$ FALSE $\wedge$ MeetingChairOccupy $==$ FALSE $\wedge$ $P I R==F A L S E)$ confirms Activity 5-Absence. The condition- (1 to 5 ) is then used in our recognition algorithm represented in Algorithm 1 1 To give best performance, synchronization of the sensed data is important, the more precise the alignment of the reports is, the more accurate the sensor fusion is. We use the Packet-level Time Synchronization that is limited to single-hop communication described in (TEP133, 2008). More precisely, the proposed recognition algorithm is given in Algorithm 1 .

In the algorithm, the input is a vector of sensor data, in our case, it is $V=$ [WorkingChairOc-

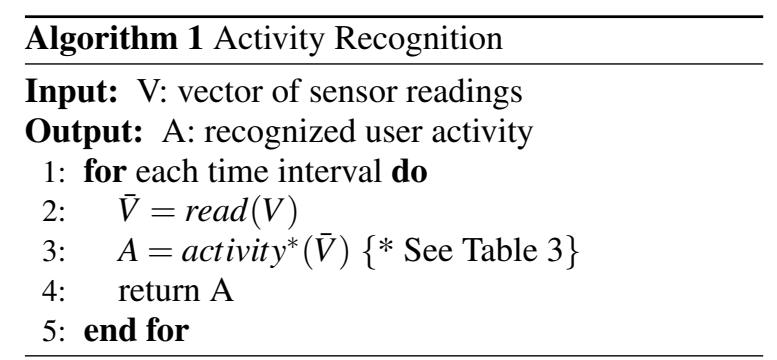

cupy, Key/MouseOccupy, MeetingChairOccupy, $\mathrm{Hu}$ manVoice, PIR]. Data is pushed to the Base Station periodically and the vector $V$ is updated with the data and the time stamp (e.g. each minute, each five minutes, etc.). The implemented time synchronization mechanism (Packet-level Time Synchronization) guarantees sensor data timestamps are accurate up to $\pm\left(T_{r} / 2-\min \right)$ (where $T_{r}$ is the round trip time and min is an estimated minimum time for sending any message). For a single hop network, the accuracy is very high, given the small value of $T_{r}$, especially when compared with the size of the time interval. By using reliable messaging, the base station is also guaranteed to eventually get all sensor readings for each time interval; moreover, message omissions due to packet losses are handled by keeping the previous state as valid until a new message arrives. The algorithm works as an infinite cycle that at the end of each time interval reads all the pushes of the sensor data and it uses them to determine which activity has taken place in that time interval, that is, the sensor data is read to $\bar{V}$ from $V$ by $\bar{V}=\operatorname{read}(V)$ and then the function activity $(\bar{V})$ recognizes the occurring activity based on the information fusion cases of Table 3 . 


\section{IMPLEMENTATION}

To test the quality of the activity recognition with real sensors, we make a study in our own office at the University of Groningen, using three types of sensors and classifying five activities, as presented in the previous sections. In one of the rooms, chairs occupancy is detected by a pressure sensor. We also place one acoustic sensor near the keyboard and mouse of desktop position. Another acoustic sensor is placed at the meeting table for detecting human voice as the sign of a meeting taking place. Finally, one central ceiling mounted PIR sensor is placed in the center of the room. Figure 6(a) overviews our instrumented room.

\subsection{Simple Sensors}

We use IEEE 802.15.4 compliant wireless sensors based on the original open-source "TelosB" platform design developed and published by the University of California, Berkeley ("UC Berkeley"). The hardware is produced by Advantic Systems (ADV, 2011), see the photos in Figure 4 The sensors are equipped with ultra low-power 16bit microcontroller MSP430 and run a low-power consumption management algorithm. The motes also have an extension interface that can be used to connect various sensor boards containing photo-, temperature-, humidity-, pressure sensors, and accelerometers, magnetometers and microphones. The on-board PIR and Microphone are used together with FlexiForce pressure sensor. The motes are programmed in nesC and run on the TinyOS 2.1.1 platform (Philip Levis, 2004), a lightweight, low-power platform for embedded operating systems. The component for Information Fusion and Activity Recognition on the Base Station is written in Java. Let us consider the sensors one by one.

\subsubsection{Pressure Sensor}

We use a pressure sensor to detect chair occupancy status. The sensor designed by Advantic Systems uses the Tekscan ${ }^{\circledR}$ A201-100 FlexiForce ${ }^{\circledR}$ sensor (see Figure 4(b), which provides force and load measurements. The FlexiForce sensor can be used to measure both static and dynamic forces (up to $1001 \mathrm{~b}$ or $400 \mathrm{~N}$ ), and is thin enough to enable non-intrusive measurement. In our experiments, the sensor is placed on the chair as shown in Figure 6(c) Figure 2 shows a 30minute (from minute 28 to minute 55 ) result from one of our tests about chair pressure. When no one sits on the chair, pressure sensor returns a value which is less than 5 Newtons and has an average value of about 1 Newton. While the chair is occupied, the value of pressure is greater than 20 Newtons and can go up to almost 100 Newtons. Additionally, when working at the desk (with or without PC), the user might stand up for a while or change the sitting posture thus the pressure value hovers over time, however, we establish from experimental data that when the chair is occupied the pressure average value in one minute is greater than 20 Newtons while less than 10 Newtons is the value when the chair is unoccupied. Therefore, a one-minute average value of 20 Newtons is set as the threshold to distinguish among the two states of the chair's occupancy. In order to get a precise average value, it is calculated from 10 polled values every minute. The average value is then compared with the threshold (20 Newtons) to identify the chair status. The pressure sensor node wirelessly sends chair status packets with a single value (TRUE if it detects chair occupy; FALSE otherwise) to the Base Station.

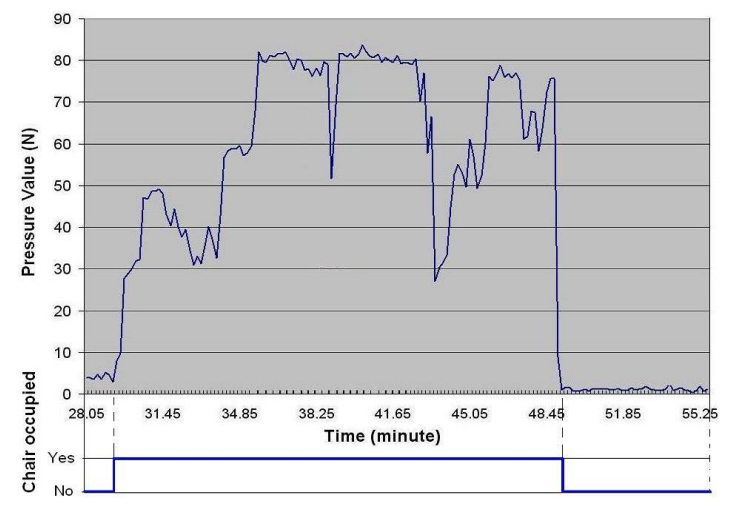

Figure 2: Pressure Sensor Data

\subsubsection{PIR Motion Sensor}

The Passive InfraRed sensor detects the motion of users inside the office. The sensor uses the Perkin Elmer Optoelectronics ${ }^{\circledR}$ LHI878 sensor, Figure 4(c) The LHI878 pyroelectric infrared-detector series are the standard dual element design recommended for all variants of motion control. PIR sensor data provides indication of whether the user is likely to be in the office, especially when all other sensors do not detect any activity. Different from pressure information, with which average value reflects the pressure during the polled period, user movement only triggers PIR sensor for short time intervals. Figure 3 illustrates a 10-minute reading (from minute 45 to minute 55) of PIR data. PIR value is measured in analog-to-digital converter (ADC) unit. When there is no movement inside the field of view, the used PIR sensor returns a small value that stably is either one, two or three ADC. Movement is detected when PIR is triggered and it returns a value that is also stably at around 
2840 ADC. Based on these experimental results we use $2800 \mathrm{ADC}$ as the threshold for detecting motion of user inside the office. After the polled period, maximal PIR value is taken, instead of calculating an average value, as the key value to compare with the threshold of 2800 ADC. The PIR sensor also sends a single-value status (TRUE or FALSE) packet to the base station each minute.

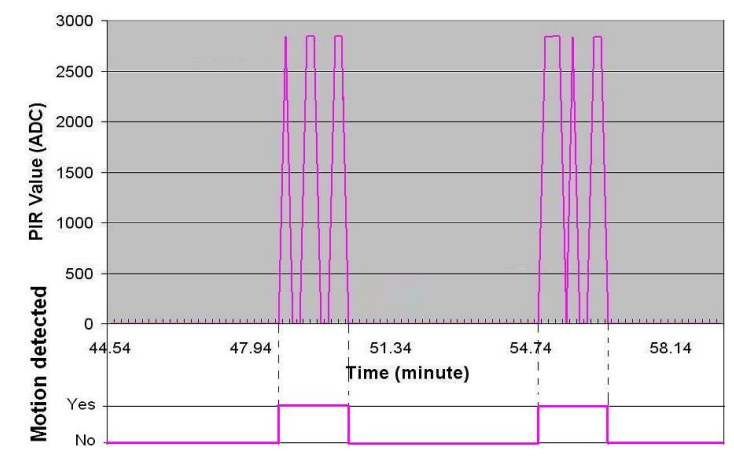

Figure 3: PIR Sensor Data

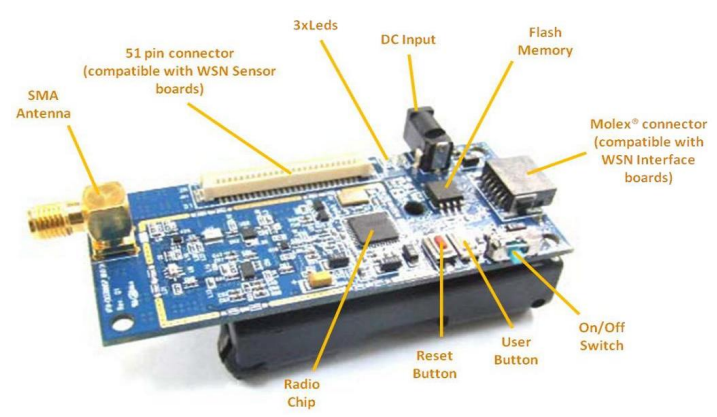

(a) Advantic TelosB mote

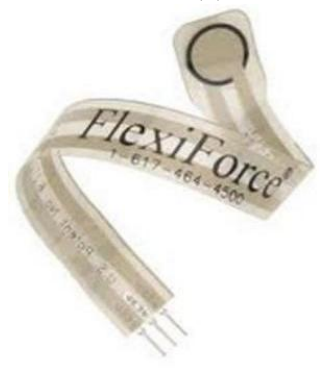

(b) Tekscan ${ }^{\mathbb{R}}$ A201-100

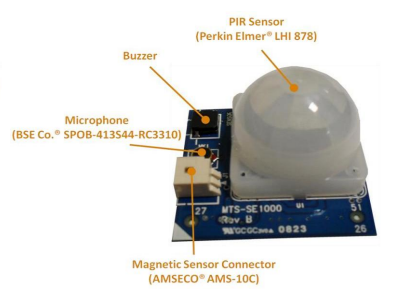

(c) PIR and Acoustic
Figure 4: Advantic Sensor. Retrieved November 112011 from http://www.advanticsys.com/

\subsubsection{Acoustic Sensor}

The SE1000 acoustic sensor provided by Advantic Systems goes on the attachable sensor board is a minimicrophone (20 $16000 \mathrm{~Hz}$, SNR $58 \mathrm{~dB})$, Figure 4(c). capturing ambient audio information. It is designed to detect the presence/absence of sound.

Different from PIR or pressure data, with which we are able to set an absolute value as a threshold to distinguish occupancy states, the changes in sound level (decibel) depend on offices and settings. For example, background noise in different offices or at different time of the day is different. Therefore, the threshold value can not be given as an absolute value. However, monitored sounds (keyboard typing, mouse clicking, person speaking) make a significant change in sound level in comparison with ambient background noise. For this reason, before final deployment, one runs a training phase to establish the difference in decibel level of acoustic data between two cases. Acoustic data is sampled at $1 \mathrm{MHz}$ providing microsecond time resolution needed, in turn, for capturing the appearance of expected sounds. Then, average value of 1-minute polling data is used as decibel value of the background noise, then again, the maximal value of polled data is compared with this ambient noise's level to check the appearance of monitored sounds.

For sensing keyboard/mouse sounds, we place the acoustic sensor within 30 centimeters from the keyboard and mouse, as shown in Figure 6(b), another acoustic sensor is placed on the meeting table to sense human voice. In case of keyboard typing and/or mouse clicking, data from early experiments shows that when keyboard is being typed and/or mouse is being clicked the change in decibel level is greater than $20 \mathrm{~dB}$. In "person speaking" case, the difference between background noise and human voice is even bigger, the subtraction of average value from maximal one leaves a value greater than $30 \mathrm{~dB}$. Figure 5 shows results from some of our experiments during training phase, illustrating 1-minute polling. Figure 5(a) exemplifies the result from Key/Mouse usage while Figure 5(b) represents result from Meeting table when person's speaking sound is present. From the results during the training phase, we set the value of $20 \mathrm{~dB}$, getting from the subtraction of average value from maximal one as the threshold for both keyboard/mouse acoustic sensor at the working table and human voice acoustic sensor at the meeting table.

\subsection{Experimental Setup and Results}

Using the layout of Figure 6 the sensors just described and the classification algorithm presented in Section 2, we perform an experiment lasting 5 days, that is, daily from 9:00 AM to 18:00 PM, from August 22 to 26,2011 to verify the accuracy of the proposed 


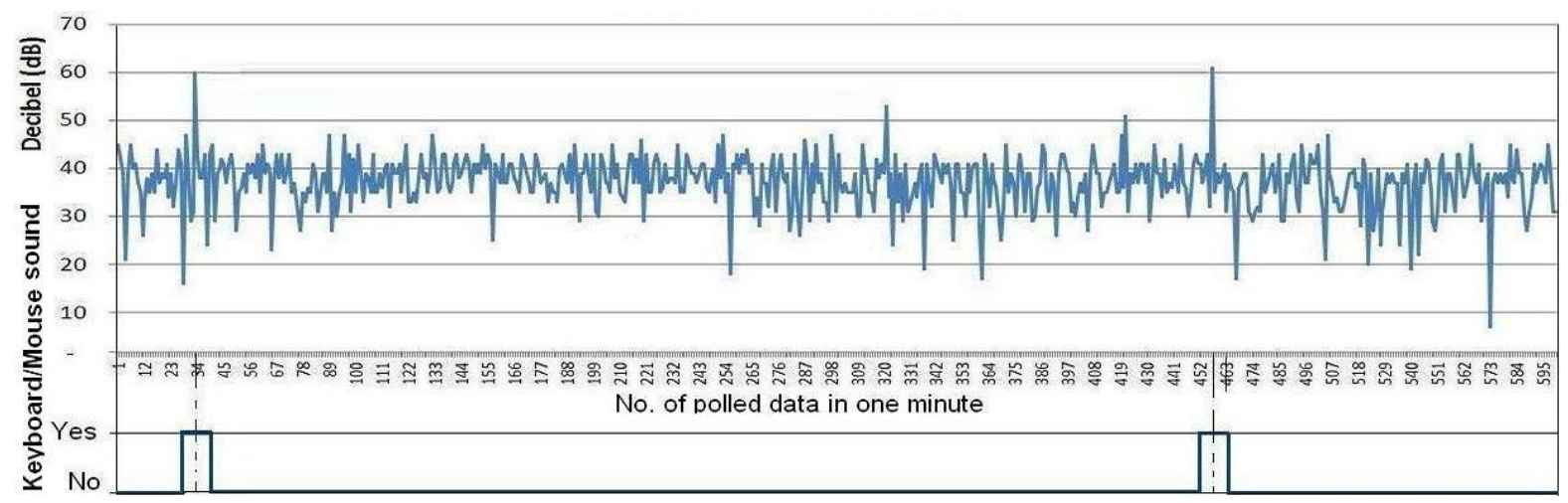

(a) Keyboard/Mouse Acoustic Data

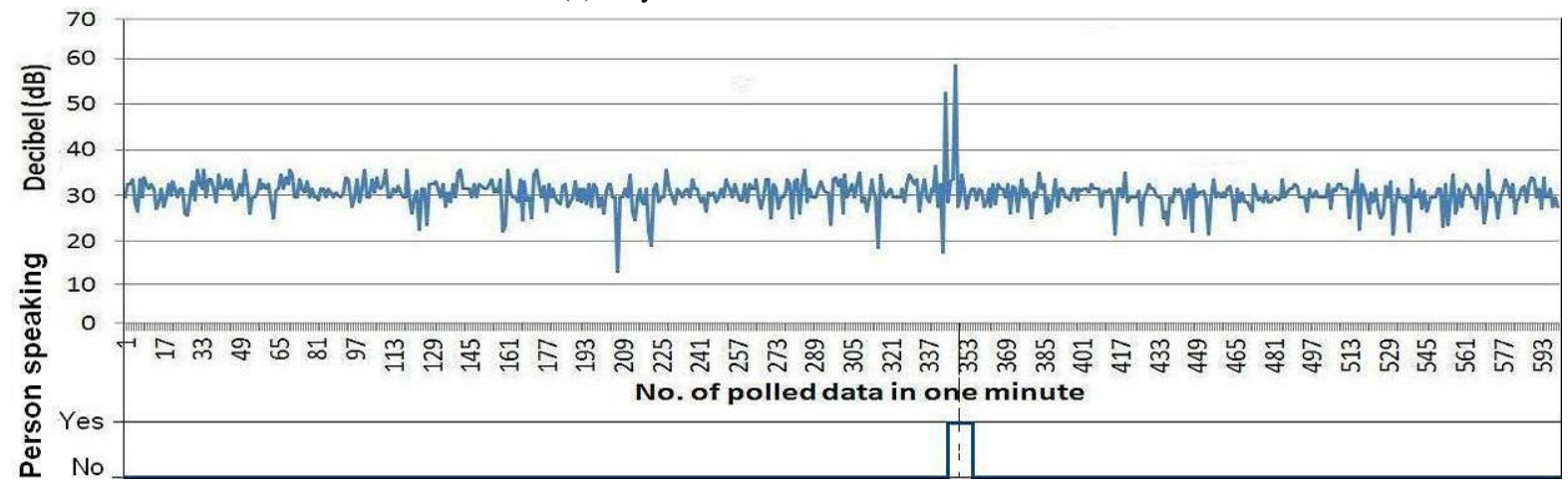

(b) Human Voice Acoustic Data

Figure 5: Acoustic Data

approach in terms of activity classification. During the experiment, the user takes accurate notes of actual activities happening in the office every five minutes, which is used as golden truth for the evaluation. Table 4 shows the real occurrences of activities for each minute of each activity.

Table 4: Golden Truth of activities' happening time

\begin{tabular}{|c|c|c|c|c|c|}
\hline Activity & $\begin{array}{c}\text { Working } \\
\text { with } \\
\text { PC }\end{array}$ & $\begin{array}{c}\text { Working } \\
\text { without } \\
\text { PC }\end{array}$ & $\begin{array}{c}\text { Having } \\
\text { a } \\
\text { meeting }\end{array}$ & Presence & Absence \\
\hline $\begin{array}{c}\text { Happening } \\
\text { time (min.) }\end{array}$ & 1350 & 595 & 180 & 265 & 310 \\
\hline Total (min.) & \multicolumn{5}{|c|}{$\mathbf{2 7 0 0}$} \\
\hline
\end{tabular}

As can be seen, the user spends most of his/her time Working with PC, 1350 out of 2700 minutes, followed by Working without PC with 595 minutes over the experiment. Another 180 minutes during one working week is spent for Having a meeting activity while Presence and Absence activities take 265 and 310 minutes, respectively.

To be more precise, we adapt to our case the following performance measures. Given an activity A, we measure accuracy in the following way:

- FP (False Positive): the number of happening minutes of other activities recognized incorrectly as $\mathrm{A}$.

- FN (False Negative): the number of happening minutes of $\mathrm{A}$ recognized falsely as not $\mathrm{A}$.

- TP (True Positive): the number of happening minutes of A recognized truly as A.

- Recall: the proportion of the time correctly recognized as A against the real happening time of A. Also called "True Positive Rate" - the percentage of positives the system recognizes correctly.

$$
\text { Recall }=\frac{T P}{T P+F N}
$$

- Precision: the rate of the real happening time of $\mathrm{A}$ over all the time recognized as A.

$$
\text { Precision }=\frac{T P}{T P+F P}
$$

The overall success rate of the system is computed based on the following formula:

$$
\text { success rate }=\left(1-\frac{\sum_{\forall a c t i v i t i e s}(F P+F N)}{2700}\right)
$$




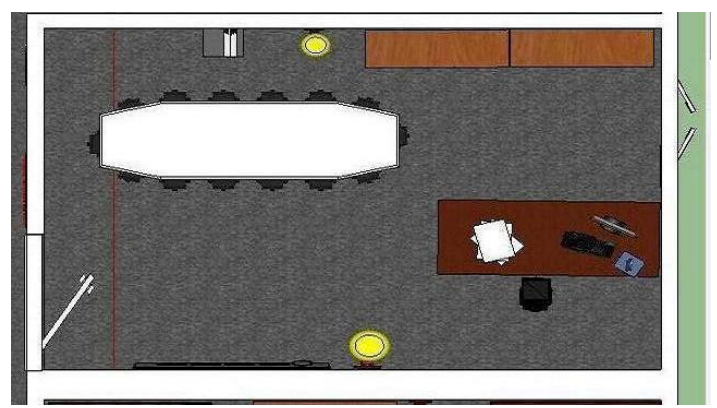

(a) Overview of the instrumented room

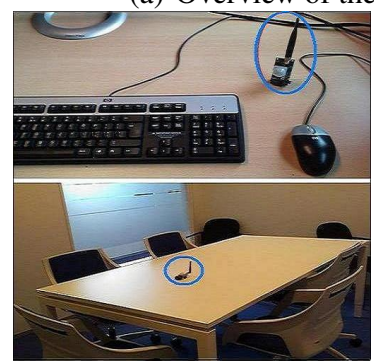

(b) Acoustic Sensors' Placement

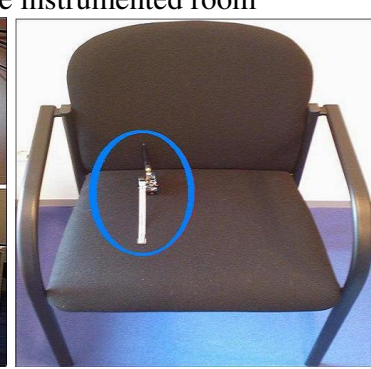

(c) Pressure Sensor Placement

Figure 6: The instrumented room

in which, $\sum_{\forall a c t i v i t i e s}(F P+F N)$ is the total minutes of wrong recognition. 2700 is the number of minutes of total experimental period.

Our system makes a classification every minute. The results of our experiment are presented in Table 5. The results for TP of Working with PC is 1350 out of 270 minutes. The FN is kept to 0 minutes, which leads to the perfect Recall of $100 \%$ while the Precision is $96.84 \%$ caused by 44 minutes of the FP. Our system recognize 565 minutes for Working without $P C$ activity with 30 minutes of FN and the FP is 0 , resulting in $94.96 \%$ of Recall and $100 \%$ of Precision. The next row in the table depicts the atributes of Having a meeting activity. $93.57 \%$ is the Precision of the system as recognized happening time is 192 minutes while the real one is 180 minutes. As a consequence, the system recognizes mistakenly 12 minutes more than the total happening time of this activity, reflected on FP is $12, \mathrm{FN}$ is 0 , and Recall for the one is $100 \%$. The Recall of the system in recognizing Presence and Absence activities are $94.34 \%$ and $96.45 \%$, respectively. The FN values express that 15 minutes for Presence and 11 minutes for Absence are recognized incorrectly as the activities. However, the Precision for both of them is $100 \%$.

\subsection{Discussion}

By analyzing raw result data, we find out that 44minute FP of "Working with PC" is taken from FNs

Table 5: Experimental Results

\begin{tabular}{|l|c|c|c|r|r|}
\hline Activity & $\begin{array}{c}\text { FN } \\
(\mathbf{m i n} .)\end{array}$ & $\begin{array}{c}\text { FP } \\
(\mathbf{m i n} .)\end{array}$ & $\begin{array}{c}\text { TP } \\
(\mathbf{m i n} .)\end{array}$ & $\begin{array}{r}\text { Recall } \\
(\mathbf{\%})\end{array}$ & $\begin{array}{r}\text { Precision } \\
(\mathbf{\%})\end{array}$ \\
\hline Working with PC & 0 & 44 & 1350 & 100 & 96.84 \\
\hline Working without PC & 30 & 0 & 565 & 94.96 & 100 \\
\hline Having a meeting & 0 & 12 & 180 & 100 & 93.75 \\
\hline Presence & 15 & 0 & 250 & 94.34 & 100 \\
\hline Absence & 11 & 0 & 299 & 96.45 & 100 \\
\hline
\end{tabular}

of "Working without PC" and "Presence" activities. While 12-minute FP of "Having a meeting" is taken from FNs of "Presence" and "Absence" activities. This is caused by inaccurate acoustic data decisions. At some moments some louder sound appears causing the subtraction from the average value and thus brining the maximum value above the $20 \mathrm{~dB}$ threshold, thus the acoustic sensors returns TRUE. As a consequence, the system indicates the activity as "Working with PC" or "Having a meeting".

The error may cause unnecessary energy usage by increasing the PC-related or meeting-related load (e.g. LCD monitor or lights are not turned off). Meanwhile, this results in the Recall of "Working with PC" and "Having a meeting" activities is $100 \%$, making sure that $\mathrm{PC} /$ meeting-related devices are working probably while the user is really working with the PC or having a meeting. These satisfy one of the important criterion of a smart building that is the user comfort has higher priority than energy saving.

\section{RELATED WORK}

In recent years, many research efforts have been carried out to design smart building. The advent of low-cost wireless sensor networks has enabled wider deployment opportunities of a large number of connected sensors thus allowing for improved sensing in buildings. We shall review here some of the most relevant previous works on using activity recognition as a driver for control of smart building with respect to energy-aware perspective.

Prior researches in HVAC control systems show that occupancy information can be used to drive a more optimized HVAC schedule. In (Erickson and Cerpa, 2010) or (Agarwal et al., 2010), the authors propose a demand response HVAC control strategy that uses real-time occupancy monitoring with occupancy prediction to achieve efficient conditioning. Another example is (Newsham and Birt, 2010), where the ARIMAX model is developed to forecast the power demand of the building in which a measure of 
building occupancy is a significant independent variable and increased the model accuracy. However, due to the difficulty in obtaining real-time accurate occupancy data, many of these techniques focus on using pre-determined schedules. Furthermore, these researches only try to use occupancy data, far less than the details of user activity.

In the context of Artemis SOFIA Project (SOFIA, 2009), the authors in (Niezen et al., 2010) provide a way for users to physically interact with devices, expressing themselves. Through developing meaningful interaction devices their system can predict to understand what the user is trying to accomplish, nevertheless user needs to perform necessary actions. Our approach differs in trying to recognize user's activity automatically.

Simple sensors are used in many modern buildings. For instance, Passive InfraRed (PIR) based sensors are often used (especially with lighting system) for occupancy detection. The sensors are connected directly to local lighting fixtures. These PIR sensors are also simple movement sensors and often cannot actually determine if the room is occupied or not. Thus, most of them use a timeout for shutting off the lights (30 minutes is common) which can be sub-optimal. (Padmanabh et al., 2009) investigate the use of microphones and PIR sensors for the efficient scheduling of conference rooms. (Delaney et al., 2009) use PIR based wireless occupancy sensors to measure wasted energy in lighting even when there are no occupants. In the AIM Project (Barbato et al., 2009), authors suggest to measure some physical parameters like temperature and light as well as user presence based on PIR sensors in each room of a house. (Gao and Whitehouse, 2009) seek to use coarse occupancy data (leave home, return home) to drive a self-programming home thermostat; however the focus is on the thermostat self-programming algorithms, and not on obtaining accurate occupancy. (Wang S, 1998) examine CO2-based occupancy detection yet it is very slow to detect events such as incoming people. These efforts, however, neither use occupancy information to drive actual systems nor evaluate accuracy of their detection sensor, and are rarely used for intelligent energy management in buildings. Our system uses the same simple sensors but provides far more detailed information on the activity of the users.

More advanced systems, such as using sonarbased sensing (Tarzia et al., 2009) or cameras and vision algorithms (Teixeira and Savvides, 2008) have been presented, though they suffer from deployability, cost and privacy issues. SCOPES (Kamthe et al., 2009) is an occupancy monitoring system that detects near real-time occupant movement between rooms with an accuracy of $80 \%$. (Erickson et al., 2009) propose a wireless network of cameras (which have the aforementioned privacy and cost issues) to determine real-time occupancy across a larger area in a building, focusing more towards coarse-grained floor-level occupancy detection. In (Singhvi et al., 2005), the occupants are equipped with sensor badges, with which it is possible to achieve relatively accurate localization using, for example, RFID tags. In SPOTLIGHT (Kim et al., 2008), the authors present a prototype system that can monitor energy consumption by individuals using a proximity sensor. While the authors tackle the right challenge, the system either requires users to carry active RFID tags or to explicitly tap tags on RFID readers, which is cumbersome. In contrast, we use simple binary sensors.

In summary, most related work either uses rich sensors (e.g. cameras, RFID tags, wearable sensors) or simple sensors but providing coarse-grained occupancy information rather than detailed activities into appliance control strategies. In this paper, our system provides a finer grained activity recognition solution with simple sensors exhibiting very good classification results.

\section{CONCLUSIONS}

We believe that one of the key inputs for BAS is detailed and precise user activity information that, in turn, can drive the control of buildings and decrease energy consumption while preserving the user comfort. Our proposed activity recognition approach is useful foraccurate activity recognition at the level of individual offices. By using low-cost, binary, and wireless sensors we are able to recognize five office activities for one person in a standard office room. Through initial experiments, we show that the solution can recognize user activity accurately with a success rate of $94.81 \%$. More importantly, falsenegatives (which may lead to discomfort) are kept perfectively at $0 \%$, satisfying user expectations at work. These promising results suggest to investigate further the office activity recognition with simple sensors. In particular, it is important to enrich the set of recognized activities, possibly using other types of simple sensors. It is also essential to consider multi-user in one room and multi-room situations. Finally, we plan to couple approaches, as the one presented here, with actual building control, to measure the amount of actually saved energy and test the resulting user experience, this in the context of the GreenerBuildings project. 


\section{ACKNOWLEDGEMENT}

We thank Peter Kamphuis, Jeroen Jager and Dimitri de Jong for providing data on the survey reported in Section 2. We also thank Sharique Arshi for useful discussions on acoustic sensing. Tuan Anh Nguyen is supported by the Vietnam International Education Development program (VIED). The work is supported by the EU FP7 Project GreenerBuildings, contract no. 258888 and the Dutch National Research Council under the NWO Smart Energy Systems program, contract no. 647.000.004.

\section{REFERENCES}

ADV (2011). Advantic Systems website http://www. advanticsys.com/.

Agarwal, Y., Balaji, B., Gupta, R., Lyles, J., Wei, M., and Weng, T. (2010). Occupancy-driven Energy Management for Smart Building Automation. In Proceedings of the 2nd ACM Workshop on Embedded Sensing Systems for Energy-Efficiency in Building, BuildSys '10, pages 1-6, New York, NY, USA. ACM.

Barbato, A., Borsani, L., Capone, A., and Melzi, S. (2009). Home Energy Saving through a User Profiling System based on Wireless Sensors. In Proceedings of the First ACM Workshop on Embedded Sensing Systems for Energy-Efficiency in Buildings, BuildSys '09, pages 49-54, New York, NY, USA. ACM.

Delaney, D. T., O'Hare, G. M. P., and Ruzzelli, A. G. (2009). Evaluation of Energy-efficiency in Lighting Systems using Sensor Networks. In Proceedings of the First ACM Workshop on Embedded Sensing Systems for Energy-Efficiency in Buildings, BuildSys '09, pages 61-66, New York, NY, USA. ACM.

EPBD (2002). European Union Directive on the Energy Performance of Buildings - 2002-91-E.

Erickson, V. L. and Cerpa, A. E. (2010). Occupancy based Demand Response HVAC Control Strategy. In Proceedings of the 2nd ACM Workshop on Embedded Sensing Systems for Energy-Efficiency in Building, BuildSys '10, pages 7-12, New York, NY, USA. ACM.

Erickson, V. L., Lin, Y., Kamthe, A., Rohini, B., Surana, A., Cerpa, A. E., Sohn, M. D., and Narayanan, S. (2009). Energy Efficient Building Environment Control Strategies using Real-time Occupancy Measurements. In Proceedings of the First ACM Workshop on Embedded Sensing Systems for Energy-Efficiency in Buildings, BuildSys '09, pages 19-24, New York, NY, USA. ACM.

Gao, G. and Whitehouse, K. (2009). The Self-programming Thermostat: Optimizing Setback Schedules based on Home Occupancy Patterns. In Proceedings of the First ACM Workshop on Embedded Sensing Systems for Energy-Efficiency in Buildings, BuildSys '09, pages 67-72, New York, NY, USA. ACM.

GreenerBuildings (2010). GreenerBuildings Project website http://www.greenerbuildings .eu/
Kamthe, A., Jiang, L., Dudys, M., and Cerpa, A. (2009). SCOPES: Smart Cameras Object Position Estimation System. In Proceedings of the 6th European Conference on Wireless Sensor Networks, EWSN '09, pages 279-295, Berlin, Heidelberg. Springer-Verlag.

Kim, Y., Charbiwala, Z., Singhania, A., Schmid, T., and Srivastava, M. B. (2008). Spotlight: Personal Natural Resource Consumption Profiler. HotEmNets 2008.

Newsham, G. R. and Birt, B. J. (2010). Building-level Occupancy Data to Improve ARIMA-based Electricity Use Forecasts. In Proceedings of the 2nd ACM Workshop on Embedded Sensing Systems for EnergyEfficiency in Building, BuildSys '10, pages 13-18, New York, NY, USA. ACM.

Niezen, G., Hu, J., and Feijs, L. M. G. (2010). From Events to Goals: Supporting Semantic Interaction in Smart Environments. In 1st Workshop on Semantic Interoperability for Smart Spaces (SISS2010).

Padmanabh, K., Malikarjuna, V, A., Sen, S., Katru, S. P., Kumar, A., C, S. P., Vuppala, S. K., and Paul, S. (2009). iSense: a Wireless Sensor Network based Conference Room Management System. In Proceedings of the First ACM Workshop on Embedded Sensing Systems for Energy-Efficiency in Buildings, BuildSys '09, pages 37-42, New York, NY, USA. ACM.

Philip Levis, Sam Madden, J. P. R. S. A. W. D. G. J. H. M. W. E. B. D. C. (2004). TinyOS: An Operating System for Sensor Networks. In in Ambient Intelligence. Springer Verlag.

Ploetz, T. and Olivier, P. L. (2010). How To Do Good Research In Activity Recognition - Position Paper. In How To Do Good Research In Activity Recognition. Workshop in conjunction with Pervasive 2010.

Singhvi, V., Krause, A., Guestrin, C., Garrett, Jr., J. H., and Matthews, H. S. (2005). Intelligent Light Control using Sensor Networks. In Proceedings of the 3rd international conference on Embedded networked sensor systems, SenSys '05, pages 218-229, New York, NY, USA. ACM.

SOFIA (2009). Artemis SOFIA website http://www. sofia-project.eu/

Tarzia, S. P., Dick, R. P., Dinda, P. A., and Memik, G. (2009). Sonar-based Measurement of User Presence and Attention. In Proceedings of the 11th international conference on Ubiquitous computing, Ubicomp '09, pages 89-92, New York, NY, USA. ACM.

Teixeira, T. and Savvides, A. (2008). Lightweight People Counting and Localizing for Easily Deployable Indoors WSNs. volume 2, pages 493-502.

TEP133 (2008). TinyOS Enhancement Proposals 133: Packet-level Time synchronization.

Wang S, J. X. (1998). CO2-Based Occupancy Detection for On-Line Outdoor Air Flow Control. volume 7, pages 165-181. 